\title{
Antropalogín
}

\section{Relaciones de poder en los Hospitales. Enfermeras y padres y madres inmigrados}

\section{Power relations in Hospitals. Nurses and immigrant fathers and mothers}

Relações de Poder em hospitais. Enfermeiros e pais e mães imigrantes

Juan Luis González Pascual ${ }^{1}$ y Manuel Moreno Preciado²

${ }^{1}$ Enfermero. Licenciado en Antropología Social y Cultural. Profesor ayudante Universidad Europea de Madrid.

${ }^{2}$ Enfermero. Doctor en Antropología Social y Cultural. Profesor titular Universidad Europea de Madrid

Cómo citar este artículo en edición digital: González Pascual, J.L. y Moreno Preciado, M. (2014) Relaciones de poder en los Hospitales. Enfermeras y padres y madres inmigrados. Cultura de los Cuidados. (Edición digital) 18, 40.

Disponible en: <http://dx.doi.org/10.7184/cuid.2014.40.8>

Correspondencia: Universidad Europea de Madrid. Edificio A. Departamento de Enfermería. c/ Tajo, s/n. 28670 Villaviciosa de Odón (Madrid).

Correo electrónico: juanluis.gonzalez2@uem.es

Recibido: 12/06/2014; Aceptado: 16/09/2014

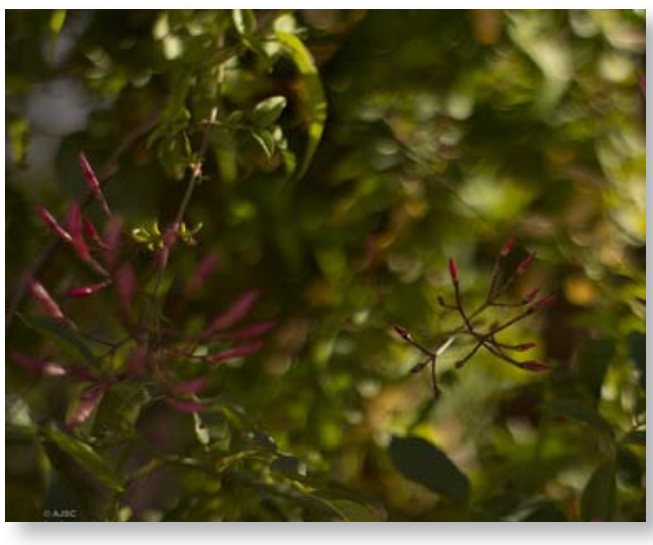

\section{ABSTRACT}

The practice of nursing takes place in the context of healthcare institutions, being the interpersonal relationship the backbone of the practice. In the case of immigrants the goal is to provide culturally competent care. However, the hierarchical and normative hospitals (total institutions) make the relationship between nurses and patients difficult, especially when they come from other cultural backgrounds. The objective of this study is to explore the power relations and to identify barriers in the relationship between nurses- immigrant fathers/mothers due to institutional constraints of the hospital. The ethnographic study collected data through the testimonies of the professionals and the parents of immigrant children and also by observing the practice environments in two public hospitals of average size of the Community of Madrid. The results show that nurses, through the exercise of institutional power, establish with immigrant parents asymmetrical relationships favored by language barriers, prejudice and stereotypes. To provide culturally competent care, you need to overcome institutional and personal barriers and establish dialogic relationships that allow flexible and individualized care, avoiding ethnocentrism.

Keywords: International migration (Emigration and Immigration), Nursing care, Nurse-patient relationship (Nurse-Patient Relations), Power. 


\section{RESUMO}

A prática de enfermagem tem lugar no contexto das instituições de saúde, com o relacionamento interpessoal o principal foco da prática. No caso dos imigrantes a meta é a prestação de cuidados culturalmente competente. No entanto, os hospitais hierárquicos e normativos (instituições totais) faz com que a relação entre enfermeiros e pacientes, especialmente quando eles vêm de outras origens culturais. O objetivo deste estudo é explorar as relações de poder e identificar barreiras no relacionamento enfermera-padre/madre imigraram devido a restrições institucionais do hospital.

Estudo etnográfico, os dados coletados através do testemunho dos profissionais e os pais de filhos de imigrantes e também observando os ambientes favoráveis à prática em dois hospitais públicos de tamanho médio da Comunidade de Madrid.

Os resultados mostram que os enfermeiros, através do exercício do poder institucional, estabelecida com mães imigrantes e pais de relações assimétricas, favorecidos por barreiras linguísticas, preconceitos e estereótipos. Para prover um cuidado culturalmente competente, é preciso superar as barreiras institucionais e pessoais e estabelecer relações dialógicas que permitem atendimento flexível e individualizado, evitando o etnocentrismo.

Palavras-chave: Migração internacional, Cuidados de enfermagem, Relações Enfermeira-paciente, Poder

\section{RESUMEN}

La práctica de la enfermería tiene lugar en el marco de las instituciones sanitarias, siendo la relación interpersonal el eje principal de dicha práctica. En el caso de los inmigrados se pretende proporcionar cuidados cultural- mente competentes. Sin embargo, el carácter jerárquico y normativo de los hospitales (instituciones totales) dificulta la relación entre enfermeras y pacientes, máxime cuando estos provienen de otros horizontes culturales. El objetivo de este estudio es explorar las relaciones de poder e identificar las barreras en la relación enfermera-padre/madre inmigrado debido a los condicionantes institucionales del hospital. El estudio, de corte etnográfico, recoge datos a través de los testimonios de los profesionales y de los padres y madres de los niños inmigrados y también mediante observación de los entornos de las prácticas en dos hospitales públicos de tamaño medio de la Comunidad de Madrid. Los resultados muestran que las enfermeras a través del ejercicio del poder institucional establecen con las madres y padres inmigrados relaciones asimétricas favorecidas por la barrera idiomática, los prejuicios y los estereotipos. Para proporcionar cuidados culturalmente competentes, es necesario superar las barreras institucionales y personales y establecer relaciones dialógicas, que permitan flexibilizar e individualizar los cuidados, evitando el etnocentrismo.

Palabras clave: Migración internacional, Atención de enfermería, Relación EnfermeraPaciente, Poder

\section{INTRODUCCIÓN}

La inmigración ha sido un fenómeno relativamente novedoso en España en las últimas décadas. De ser históricamente un país de emigrantes se pasó a recibir a inmigrantes económicos en gran número (Cabré, Domingo y Menacho, 2002), especialmente desde el año 2000 hasta comienzos de la crisis económica mundial en 2008 (Huesca, 2010). A pesar de la crisis, en los últimos cinco años el 
número de personas inmigradas se ha mantenido relativamente estable, sin descensos bruscos, representando en el año 2013 el 14\% de la población en España y el 18,9\% en la Comunidad de Madrid (Instituto Nacional de Estadística, 2013), debido, entre otras razones, a que sigue existiendo una diferencia importante entre la renta económica y la calidad de vida en España y la de sus países de origen (Cebrián, Bodega, Martín-Lou y Guajardo, 2010; Mahía, 2010).

La inmigración ha supuesto un gran cambio social en España. Los inmigrados tienen un papel protagonista en el crecimiento demográfico, dado que contribuyen a una gran parte de los nuevos nacimientos, mientras que la sociedad española actual envejece (Durán, 2011). En el año 2012 el 23\% de los nacimientos en España fueron de parejas en los que uno o ambos progenitores eran extranjeros (Instituto Nacional de Estadística, 2012).

Dado que la enfermería es una ciencia humana (De la Cuesta, 2010), cuya práctica tiene lugar en el marco de una relación interpersonal (Werner y Rouslin, 1996), los aspectos que tienen que ver con las relaciones humanas y el contexto son claves. Collière (1997:298) expresa que hay que "centrar a las personas en su contexto, intentando comprenderlo respecto a las costumbres, los hábitos de vida, las creencias" y que todo proceso de aplicación de cuidados (Ibíd., 284) "se inserta en un sistema de creencias y valores de los que son portadores (...) tanto las personas cuidadas como los cuidadores". En esta línea, existen diferentes

\begin{tabular}{|l|l|}
\hline \multicolumn{1}{|c|}{ Fuente de poder } & \multicolumn{1}{c|}{ Características } \\
\hline Informativo & Se ejerce a través de la argumentación. \\
\hline Recompensa & Se ejerce a través de un incentivo positivo. \\
\hline Coercitivo & $\begin{array}{l}\text { Se ejerce a través de un incentivo } \\
\text { negativo. }\end{array}$ \\
\hline Legítimo & $\begin{array}{l}\text { Se ejerce a través del derecho reconocido } \\
\text { al agente debido a la ley o la tradición. }\end{array}$ \\
\hline Experto & $\begin{array}{l}\text { Se ejerce a través de la superioridad de } \\
\text { conocimientos. }\end{array}$ \\
\hline Referencial & $\begin{array}{l}\text { Se ejerce a través de la admiración de la } \\
\text { persona hacia el agente de poder. }\end{array}$ \\
\hline
\end{tabular}

Tabla 1. Fuentes de poder y características. modelos y teorías de Enfermería que tienen en cuenta el contexto de las personas inmigradas, para ofrecer cuidados culturalmente competentes, los cuales implican "una mirada antropológica, de carácter cualitativo, que subyace de la perspectiva holística e implica un análisis enfermero específico" (Lillo y Casabona, 2006:90). No se trata de intentar conocer la cultura (o una serie de características) de cada grupo de personas, como si fuese algo fijo que todos tienen por igual, sino de desarrollar la habilidad de contextualizar a cada individuo concreto teniendo en cuenta su marco cultural (Arriagada, 2013).

La relación de las enfermeras con los padres y madres inmigrados se produce principalmente en el marco de las Instituciones Sanitarias. Según Goffman (2009), hay instituciones con gran tendencia absorbente o totalizadora, a las que denomina "instituciones totales", que programan e imponen todas las actividades diarias de las personas que están ingresadas en ellas. En los hospitales, el enfermo se convierte en protagonista pasivo de su vida, pasando a depender de la estructura y de las prioridades establecidas por la institución (Boixerau, 2008). El poder en las instituciones, según Foucault (2000), se mantiene en base a una serie de instrumentos, entre los que incluye la vigilancia jerárquica, la sanción normalizadora y el examen. Raven (2008) define el poder como el potencial de influir en las creencias, actitudes o conducta de otra persona $y$ enuncia seis fuentes de poder, que se detallan en la Tabla 1.

En el caso de las personas 
inmigradas, como explica Cachón (2007:37), la asimetría en la relación de poder puede ser aún mayor que en el caso de las personas autóctonas "Si el paciente pertenece a un ámbito cultural distinto al del profesional, en el que la explicación de la enfermedad o las prácticas terapéuticas sean radicalmente distintas a las del modelo biomédico, la asimetría en la relación es abismal". En este sentido, Martín et al. (2009) estudian las relaciones de poder durante la estancia hospitalaria de las mujeres tras el parto en España, mostrando que las enfermeras tienen un alto grado de poder sobre las decisiones tomadas por las madres, quienes asumen un rol casi pasivo, especialmente las madres inmigradas.

\section{OBJETIVOS}

- Explorar las relaciones de poder que se establecen entre las enfermeras y los padres y madres de los niños ingresados.

- Identificar las barreras en la relación enfermera-padre/madre inmigrado debido a los condicionantes institucionales del hospital.

\section{METODOLOGÍA}

Se ha utilizado la metodología cualitativa, concretamente el método etnográfico. Este trabajo se enmarca en una investigación más amplia acerca del cuidado en los hijos de personas inmigradas y su relación con el sistema sanitario de la Comunidad de Madrid El trabajo de campo se desarrolló entre noviembre de 2011 y febrero de 2013, de forma discontinua, en las Unidades de Obstetricia, Pediatría, Urgencias pediátricas y Cuidados Intensivos Neonatales de dos Hospitales de tamaño medio (269 y 406 camas respectivamente) de la Comunidad de Madrid. Se obtuvo informe favorable del Comité Ético de Investigación Clínica del Hospital Universitario Severo Ochoa.
Las técnicas utilizadas han sido la observación y la entrevista. Estas dos técnicas son complementarias entre sí. La observación permite proporcionar descripciones del investigador (perspectiva ETIC), la entrevista proporciona el punto de vista de las personas, los significados que los sujetos de estudio dan a su comportamiento (perspectiva EMIC). Asimismo, la observación permite poner el énfasis en la conexión entre los discursos y las prácticas, ayudando a captar los sentidos profundos de la interacción social al interpretarlos en sus contextos concretos (Velasco y Díaz de Rada, 2006). En los Anexos, la Tabla 2 describe los informantes a los que se hace referencia en este artículo.

El análisis se llevó a cabo mediante comparaciones constantes (Strauss y Corbin, 2002), realizando codificación abierta y axial. Se utilizó el programa informático ATLAS TI versión 6.2. En los Anexos, la Tabla 3 expone los núcleos temáticos y las categorías identificadas.

Los resultados se presentan, en el caso de las entrevistas, mediante citas textuales con el nombre ficticio del informante entre paréntesis. En el caso de la observación, se presentan los resultados redactados, con la fecha entre paréntesis.

\section{RESULTADOS}

En relación al primer objetivo, a continuación se presentan las relaciones de poder identificadas en el estudio.

- Las enfermeras actúan como representantes de la institución, garantes del cumplimiento de las normas.

Este papel está sancionado por la Institución, como se observa en el cartel de "Normas de visita" presente en uno de los Hospitales, donde dice: "Colabore con el personal del hospital y siga 
sus indicaciones" (10 de noviembre de 2011), y es percibido por parte de las enfermeras:

"Investigador: En tu opinión, como parte de tu trabajo, ¿también está el velar un poquito porque los familiares y los acompañantes cumplan las normas del hospital? Enfermera: Claro. Es un trabajo totalmente de enfermería porque eres el que estás ahí con ellos veinticuatro horas" (Fermina), "tampoco somos la autoridad ¿no?, pero somos un poco las que ponemos las normas" (Fabiola).

En el cumplimiento de ese rol, las enfermeras se quejan de que los padres y madres inmigrados incumplen más las normas y se enfrentan a ellas: "encuentro diferencias en cuanto que a ellos hacen un poco... a veces lo que quieren (...) En general los extranjeros sí que suelen... (...) saltarse más las normas (...) Los extranjeros... protestan más, se enfrentan más" (Leonor).

Las enfermeras pueden graduar la forma en que actúan como intermediarios con la institución. En un extremo estaría cuando se estandariza la atención y se actúa igual para todos, cumpliendo las normas a rajatabla, en el otro, cuando se individualiza y personaliza, flexibilizando las normas según cada persona y situación. A continuación se presentan dos respuestas ante una misma situación, que sirven como ejemplo. Durante el ingreso en la planta de Obstetricia, el personal sanitario explica a los padres que puede quedarse a dormir un acompañante en el sofá pero que tiene que traerse una sábana porque no dan en el hospital (27 de enero de 2012). Por otro lado, en la planta de Pediatría, cuando una madre pide una sábana para ella, la enfermera le dice "no nos dejan dar sábana a los acompañantes, lo hacemos bajo cuerda", pidiendo que la recoja, que la ponga solo por la noche y no por el día (24 de marzo de 2012).
- Las enfermeras sancionan los comportamientos inadecuados.

Se espera de los padres y madres que hagan caso a las instrucciones de la enfermera, $o$ pueden sufrir algún tipo de reprimenda: "saben que si las pillamos pues... claro hm... se van a llevar bronca. Bueno, bronca... que se van a llevar su regañina correspondiente" (Lara).

- Las enfermeras actúan como profesionales expertos.

Hay relaciones basadas en el rol de la enfermera como profesional que tiene un conocimiento del cual carecen los padres y madres, y que hace y enseña lo que hay que hacer según su criterio: "mientras esté ingresado el niño no va a comer un yogurt de leche de vaca, porque si al niño le da alguna reacción (...) la responsable soy yo, entonces nosotros esas cosas no las hacemos, vienen mucho pidiendo: dame un yogurt porque es que... en casa le doy yogurt, bueno, en casa es casa, nosotros aquí tenemos nuestros protocolos (...) ella le lavaba de una forma y ya fue como... el niño se moja, el niño se mete en la bañera y se va a meter en la bañera" (Fabiola).

- Las enfermeras también establecen relaciones basadas en el diálogo.

Por otro lado, hay relaciones basadas en el diálogo y la persuasión, en la que se aconseja y se deja que las personas tomen decisiones informadas: "no podemos obligar a nadie, de hecho se le respeta si... yo les puedo explicar y les puedo decir que les va a venir bien pero... en su mano está hacerlo o no hacerlo, ahí ya nosotros..." (Leonor)

En relación al segundo objetivo, a continuación se identifican y analizan las barreras en la relación enfermera - padre o madre inmigrado, relacionadas con los condicionantes institucionales. 


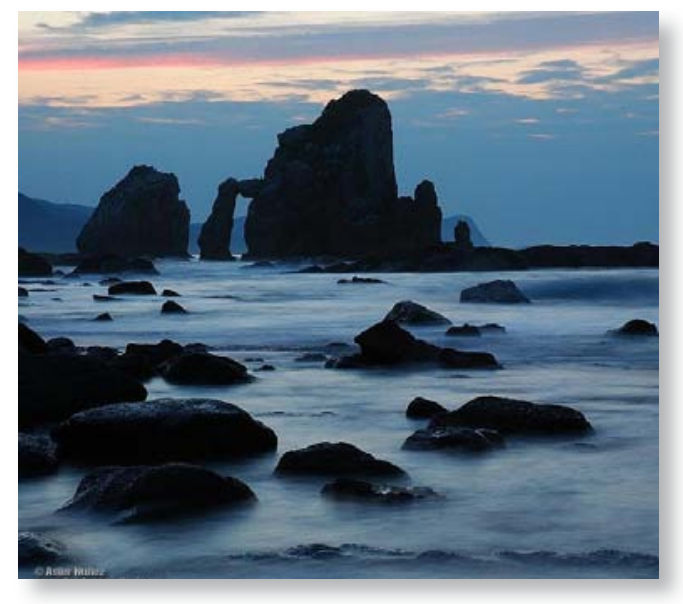

- La barrera idiomática. Está presente en muchos padres y madres inmigrados y se agrava por la ausencia de traductores en los Hospitales. Dificulta que las enfermeras puedan relacionarse con ellos igual que con el resto de padres. No se pueden explicar los cuidados hablando, dialogando: "a mí lo que me resulta más difícil es cuando hay una barrera idiomática. Yo... reconozco que me cuesta... mucho y porque muchas veces no puedes explicar lo que tú quieres explicar" (Lucrecia).

El no poder entenderse fomenta actitudes negativas de recelo o sospecha en las enfermeras, que condicionan la relación: "otros no sabes si entienden y te están tomando el pelo" (Fermina), "y la excusa yo creo que mucha la del idioma 'que es que no entiendo mucho' pues chico... lo de siempre, entendemos lo que queremos y lo que no, no" (Lorena).

- Los prejuicios y estereotipos. Las ideas preconcebidas generan conflictos de autoridad: "Algunas son un poco prepotentes con las musulmanas, y las tratan de 'estas moras'" (Basima), "me retrasé 10 minutos, me habian nombrado y luego me hicieron esperar mucho tiempo, y me dijo (...) es que vosotras las inmigradas siempre la lían, por qué no se regresan a su país" (Guadalupe).
- El etnocentrismo y la prioridad del proceso técnico. Las enfermeras refieren que las costumbres de los padres y madres inmigrados se respetan mientras que no interfieran con la seguridad de la madre, del niño o con su trabajo: "yo creo que lo que nos molesta de todas estas cosas tan arraigadas es eso, que sea perjudicial tanto para la mamá como para el bebé" (Laura), "mientras eso no... no nos afecte a nosotros a nivel enfermera o no afecte al niño eh... no hay, no hay por qué opinar sobre ello ni hay por qué hacer un mundo" (Fabiola). Pero el trabajo de la enfermera tiene prioridad, por ejemplo se entra en las habitaciones para hacer la ronda o la visita médica a pesar del cartel que informa que la madre está dando lactancia materna y que no debe interrumpirse (10 de noviembre de 2012).

- Los roles de género. La opinión de la enfermera sobre lo que es beneficioso prevalece sobre las costumbres culturales en los roles de género, como en el caso de la participación de los padres (hombres) en el método canguro (piel con piel) en las unidades Neonatales: "sobre todo es trabajar mucho con los padres el tema de que el papá les coja, porque muchos no quieren cogerle, te dicen que no se atreven, que culturalmente no les quieren coger (...) igual que un papá musulmán que no quiere coger a su hijo es decir: ven conmigo, yo te voy a ayudar, siéntate y te... yo te lo doy o cógele la mano estamos aquí, es intentar un poco... acercarlos" (Lucrecia).

\section{DISCUSIÓN}

Los resultados muestran que las enfermeras se relacionan con los padres y madres basándose en diferentes tipos de poder según la clasificación de Raven (2008). Entre otros, se ejerce poder legítimo, relacionado con el rol de 
trabajador de una Institución, asociado con el poder coercitivo, en el que se reprende al que se sale de la norma. También se ejerce poder experto, basado en la diferencia de conocimientos sobre la salud y la enfermedad que se da por supuesta debido a la profesión ejercida.

En las relaciones basadas en el poder legítimo, coercitivo y experto, se construyen relaciones asimétricas entre los profesionales sanitarios y los pacientes. Se trata de un modelo monológico (Martínez, 2008) de relación, vertical (arriba-abajo), entre quien manda y quien obedece, quien sabe y quien no sabe (o está equivocado).

El modelo opuesto es el modelo dialógico, horizontal, en donde se sitúa al mismo nivel, en pie de igualdad, el conocimiento experto y el conocimiento del paciente (Moreno, 2010), consiguiendo un "diálogo de saberes", un proceso comunicativo en el que ambos participantes tienen la intención de comprenderse a pesar de sus diferencias, y que pone en valor los conocimientos tradicionales (Bastidas et al., 2009). Este es el caso de las relaciones basadas en el poder informativo, y las situaciones en las que se ejerce el poder legítimo de forma dialogada con los padres y madres, flexibilizando y adaptando las normas de la Institución.

En el caso de los padres y madres inmigrados, son particularmente vulnerables a las relaciones monológicas. La barrera idiomática dificulta poder conversar, dialogar, tomar decisiones (Adalid, García y Alarcón, 2008) y favorece relaciones breves, en las que se dan instrucciones. Por otro lado, los estereotipos y prejuicios llevan a homogeneizar a todas las personas con unas características determinadas, como si se tratara de colectivos cerrados, olvidando la heterogeneidad existente dentro de cada grupo de origen. Se juzga a los inmigrantes desde la perspectiva (evaluada como superior) de la propia cultura, lo que se denomina etnocentrismo (Zanfrini, 2007), como en el caso de los roles de género en los hombres musulmanes. Todo ello favorece las relaciones autoritarias en las que se trata a la persona inmigrada desde la triple superioridad de ser enfermera, trabajar en la institución y ser autóctona.

Los condicionantes institucionales, tales como las normas, el sistema jerárquico (Goffman, 2009), la ausencia de traductores o la prioridad del proceso técnico, favorecen las relaciones monológicas, en las que las personas se convierten en protagonistas pasivos de su vida (Boixerau, 2008).

Para proporcionar cuidados culturalmente competentes, es necesario individualizar, huyendo de los estereotipos y prejuicios, y establecer relaciones dialógicas, basadas en el poder informativo. Hay que tener presente que el proceso de cuidados de enfermería procede de un encuentro entre dos (o varias) personas, y que cuidar es movilizar todo aquello que la persona puede hacer por si misma o con ayuda (Collière, 1997). En la atención a personas inmigradas debe estar implicado un proceso de descubrimiento y encuentro cultural, que incluye ser flexible y abierto, para aceptar diferencias y construir similitudes y para aprender de otros. Este proceso se denomina humildad cultural y es opuesto al etnocentrismo (Campinha-Bacote, 2002). El etnocentrismo puede llevar a imponer patrones culturales basados en el poder experto, como cuando se respetan las costumbres de los padres y madres inmigrados únicamente mientras no parezcan inadecuadas a la enfermera. Frente a ello, Leininger (Leininger y McFarland, 2002) establece que al prestar cuidados de enfermería se puede optar por mantener los cuidados culturales que hacen los padres o madres, negociarlos o reestructurarlos. 
Es posible proporcionar cuidados sin discriminar a nadie, de forma culturalmente competente, como expresa Plaza (2010:106) "La enfermería tiene que cuidar a sus pacientes desde la igualdad a la vez que desde la diferencia. Igualdad porque toda persona atendida tiene derecho a recibir una atención de calidad. Diferencia en cuanto a que la enfermera tiene que ser capaz de abordar a la persona desde la individualidad, valorándola de forma integral, teniendo en cuenta todos los aspectos de la persona, tanto más cuanto sus antecedentes culturales, tradicionales, geográficos, familiares o religiosos son distintos".

\section{BIBLIOGRAFÍA}

- Adalid, A.; García, M.B.; Alarcón, M.C. (2008) Superando barreras de comunicación. Paraninfo Digital [revista digital] II, 3. Disponible en: http://www.index-f.com/ para/n3/p046.php

- Arriagada, E. (2013) Historias del otro. Migración: Psicología y Literatura. Punto Rojo Libros, Sevilla.

- Bastidas, M.; Pérez, F.N.; Torres, J.N.; Escobar, G.; Arango, A.; Peñaranda, F. (2009). El diálogo de saberes como posición humana frente al otro: referente ontológico y pedagógico en la educación para la salud. Investigación y educación en enfermería 27(1): 104-111

- Boixerau, R.M. (2008) De la antropología filosófica a la antropología de la salud Herder, Barcelona.

- Cachón, E. (2007) El hospital y el paciente. Declaraciones de principios, realidad y paradojas. Index de Enfermería 16(56): 35-39

- Cabré, A., Domingo, A. y Menacho, T. (2002) Demografía y crecimiento de la población española durante el siglo XX. En Procesos migratorios, economía y personas (Pimentel, M. ed.), Cajamar, Almería pp. 121-138.

- Campinha-Bacote, J. (2002) The process of cultural competence in the delivery of healthcare services: a model of care. Journal of Transcultural Nursing 13(3): 181-184

- Cebrián, J. A., Bodega, M. I., Martín-Lou, M. A. y Guajardo, F. (2010) La crisis económica internacional y sus repercusiones en España y en su población inmigrante. Estudios Geográficos 71(268): 67-101

- Collière, M.F. (1997) Promover la vida. Edigrafos, Getafe.

- De la Cuesta, C. (2010) La investigación cualitativa y el desarrollo del conocimiento en enfermería. Texto \& Contexto Enfermagem 19(4): 762-766

- Durán, M.A. (2011) España hace treinta años, dentro de treinta años. Revista española de sociología 15: 127-134

- Fuente, J.A. (2008) Las instituciones y organizaciones sociales: un análisis sociológico. Pirámide, Madrid.

- Foucault, M. (2000) Vigilar y castigar. Siglo XXI, Madrid.

- Goffman, E. (2009) Internados. Ensayos sobre la situación social de los enfermos mentales. Amorrortu, Buenos Aires.

- Instituto Nacional de Estadística. (2012) Registro civil 2014. Disponible en: http://www.ine.es/inebmenu/ mnu_dinamicapob.htm

- Instituto Nacional de Estadística. (2013) Padrón municipal 2014. Disponible en: http://www.ine.es/inebmenu/ mnu_padron.htm

- Huesca, A. M. (2010) Panorama general de la inmigración en España. Miscelanea Comillas 68(132): 419-435

- Leininger, M.; McFarland, M.R. (2002) Transcultural nursing. Concepts, Theories, Research and Practice. McGraw-Hill, USA.

- Lillo, M.; Casabona, I. (2006) Fenómenos migratorios, competencia cultural y cuidados de salud. Cultura de los cuidados 20: 87-91

- Mahía, R. (2010) La irrupción de España en el panorama de las migraciones internacionales. Por qué llegaron, por qué siguen llegando y por qué lo seguirán haciendo. Información Comercial Española. Revista de Economía 854: 5-22

- Martín, A.; Ferrer, V.A.; Barceló, M.A.; Sequí, P.; Crespo, L.; Peñarroya,M. (2009) Relaciones de poder entre las puérperas y su entorno durante la estancia hospitalaria: relatos de alumnos/as de enfermería. Paraninfo Digital [revista digital] III, 7. Disponible en: http://www.index-f.com/para/n7/091p.php

- Martínez Hernáez, A. (2008) Antropología Médica. Teorías sobre la cultura, el poder y la enfermedad. Anthropos, Barcelona. Citado por: Moreno Preciado, M. 


\section{ᄃultura de las Cuidados}

(2010). Nuevos enfoques en el cuidado del “otro". Index de enfermería 19(2-3):167-171

- Moreno Preciado, M. (2010) Nuevos enfoques en el cuidado del “otro". Index de enfermería 19(2-3): 167-171

- Plaza, F.J. (2010) Cuidando a pacientes musulmanes. Las fronteras de la enfermería en la comunicación intercultural. Universidad de Almería, Almería.

- Raven, B.H. (2008) The bases of power and the power/ interaction model of interpersonal influence. Analyses of Social Issues and Public Policy 8(1): 1-22
- Strauss, A.; Corbin, J. (2002) Bases de la investigación cualitativa: técnicas y procedimientos para desarrollar la teoría fundamentada. Universidad de Antioquía, Medellín.

- Velasco, H.; Díaz de Rada, A. (2006) La lógica de la investigación etnográfica. Trotta, Madrid.

- Werner, A.; Rouslin, S. (1996) Teoría interpersonal en la práctica de la enfermería. Trabajos seleccionados de Hildegard E. Peplau. Masson, Barcelona.

- Zanfrini, L. (2007) La convivencia interétnica. Alianza, Madrid.

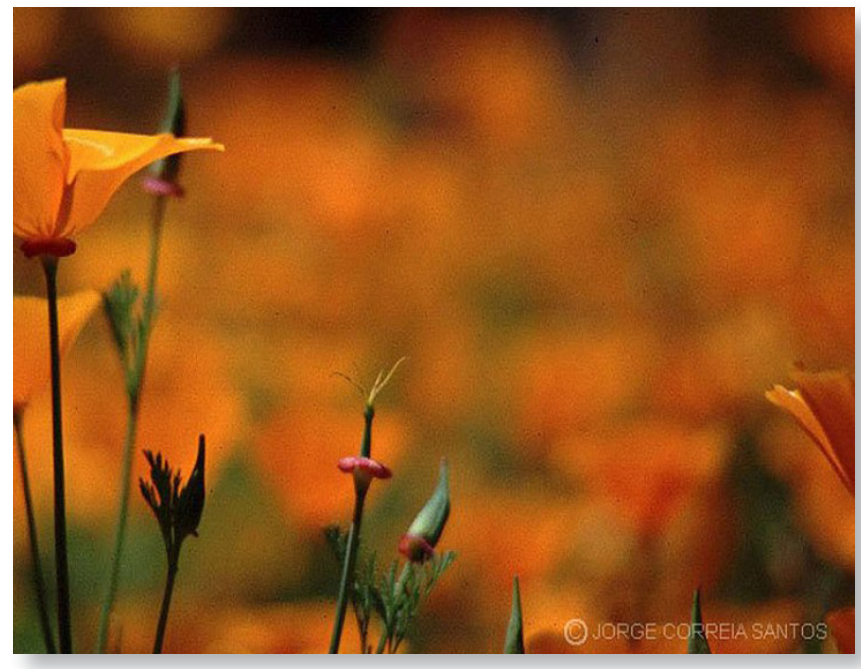

